\section{Violence, Canadian style}

With less than ten seconds left in his 'no-contact league' hockey game this past April $1^{\text {st }}$, a player from the opposing team took 7-8 strides, jumped and struck my 14-year-old son in the neck from behind. The force of the impact threw my son's stick 15 feet in the air into the stands. He fell to the ice with a concussion and a possible jaw dislocation and was transported by ambulance to the hospital where he spent the night under observation.

Hockey is the first cause of sports and recreation injuries in males, accounting for $17 \%$ of all such injuries. Although snowboarding, football, rugby, skateboarding and downhill skiing cause a greater proportion of head injuries each year, hockey injuries often result from violence and aggression, as so many front-page news items report every year. Although domestic violence, suicide and other forms of aggression are far too common in Canada, we have often taken comfort that attention-catching firearm injuries are uncommon compared to the United States. Canada may have better gun control laws and lower gun-related fatalities, but we have plenty of hockey sticks to injure our young males.

Is hockey violence a public health problem though? Is aggression an abnormal, deviant behaviour? Or, as Geoffrey Rose wrote, does the population mean predict the number of deviant individuals? ${ }^{1}$ Similar to hypertension, which is the right tail of the blood pressure distribution, Rose argued that extreme aggressive behaviours are just the tail end of a continuum of societal tolerance of violence. The distinction between abnormality and normality is a clinical imperative but it often has little basis in biology or sociology.

Punishing violent offenders may satisfy our urge for revenge, but it does little to reduce aggression in society over the long run. If the 'tail belongs to the distribution', as Rose argued, we cannot look at violence in hockey in isolation and hope for league officials to clean up their act. We must admit that it constitutes an extreme expression of our overall societal tolerance of physical, psychological, economic and social forms of aggression.

My son is now doing fine, but hockey violence and social inequalities which breed and encourage aggression are prominent in Canada. Research is needed to further our understanding of the determinants and prevention of violence in all parts of society. Rose again said it best: 'we need to accept our collective responsibility for the population's health and social well-being', p. 1034 and put in place social and economic policies to reduce inequalities and shift the entire population distribution curve of tolerance with regard to aggression.

\section{Gilles Paradis}

Interim Scientific Editor

\section{Reference}

1. Rose G, Day $S$. The population mean predicts the number of deviant individuals. Br Med J 1990;301:1031-34.
LE MOT DE LA RÉDACTION

\section{La violence à la sauce canadienne}

Le $1^{\text {er }}$ avril dernier, moins de 10 secondes avant la fin d'une partie de hockey, dans une ligue "sans contact ", un joueur de l'équipe adverse a fait sept ou huit enjambées, pris son élan et frappé mon fils de 14 ans à la nuque par derrière. Sous la force de l'impact, le bâton de mon fils a été projeté à 15 pieds dans les airs pour atterrir dans les gradins. Ayant subi une commotion et peut-être une dislocation de la mâchoire après sa chute sur la glace, mon fils a été transporté en ambulance à l'hôpital où il a passé la nuit sous observation.

Le hockey est la principale cause d'accidents du sport et des loisirs chez les hommes, où il représente $17 \%$ de ce type de blessures. La proportion des traumatismes crâniens liés au surf des neiges, au football, au rugby, à la planche à roulettes et au ski alpin augmente tous les ans, mais les blessures au hockey sont souvent le résultat d'actes de violence et d'agression, comme en témoignent de nombreuses manchettes chaque année. Bien que la violence familiale, le suicide et autres agressions ne soient que trop répandues au Canada, nous nous consolons souvent en nous disant que les blessures par balle dont on parle dans les médias sont beaucoup moins courantes ici qu'aux États-Unis. On a beau dire qu'au Canada, les lois sur le contrôle des armes à feu sont plus strictes et que ces armes causent moins d'accidents mortels, mais nous avons bien assez de bâtons de hockey pour blesser nos garçons.

La violence au hockey est-elle pour cela un problème de santé publique? Les agressions sont-elles des comportements anormaux, déviants? Ou au contraire, comme l'écrit Geoffrey Rose, la moyenne de la population permet-elle de prédire le nombre de sujets déviants ${ }^{1}$ ? Comme pour l'hypertension artérielle, qui est à l'extrémité droite de la courbe de la pression artérielle, fait-il valoir, les comportements agressifs extrêmes se situent dans le prolongement du continuum de la violence tolérée dans la société. Il est impératif, sur le plan clinique, de distinguer les comportements normaux et anormaux, mais ces distinctions n'ont souvent aucun fondement biologique ou sociologique.

Punir les délinquants violents peut assouvir notre désir de vengeance, mais à long terme, cela ne réduit pas vraiment les actes d'agression dans la société. Si les extrêmes font partie de la courbe, comme le défend Rose, il ne faut pas considérer la violence au hockey comme un cas à part en espérant que les officiels remettront de l'ordre dans la pratique du sport. Il faut admettre que cette violence est l'expression extrême de la tolérance affichée par la société en général envers les agressions physiques, psychologiques, économiques et sociales.

Mon fils s'est tout à fait remis, mais la violence au hockey et les inégalités sociales qui éveillent et encouragent les agressions sont encore bien visibles au Canada. Il faut pousser la recherche pour mieux comprendre les déterminants de la violence et les possibilités de la prévenir dans toutes les couches de la société. Comme le dit si bien Rose, "nous devons accepter que nous sommes collectivement responsables de la santé et du bien-être social de la population ${ }^{1, p .} 1034$ " et instaurer des politiques économiques et sociales visant à réduire les inégalités et à déplacer toute la courbe de répartition de la tolérance envers les agressions dans la population.

\section{Le rédacteur-réviseur scientifique intérimaire,} Gilles Paradis

\footnotetext{
Référence

1. Rose G, Day S. The population mean predicts the number of deviant individuals. Br Med J 1990;301:1031-34.
} 